\title{
Bridges with Random Length: Gamma Case
}

\author{
Mohamed Erraoui $^{1} \cdot$ Astrid Hilbert $^{2}\left[\right.$ [D $\cdot$ Mohammed Louriki $^{1}$
}

Received: 2 May 2018 / Revised: 15 March 2019 / Published online: 26 October 2019

(c) The Author(s) 2019

\begin{abstract}
In this paper, we generalize the concept of gamma bridge in the sense that the length will be random, that is, the time to reach the given level is random. The main objective of this paper is to show that certain basic properties of gamma bridges with deterministic length stay true also for gamma bridges with random length. We show that the gamma bridge with random length is a pure jump process and that its jumping times are countable and dense in the random interval bounded by 0 and the random length. Moreover, we prove that this process is a Markov process with respect to its completed natural filtration as well as with respect to the usual augmentation of this filtration, which leads us to conclude that its completed natural filtration is right continuous. Finally, we give its canonical decomposition with respect to the usual augmentation of its natural filtration.
\end{abstract}

Keywords Lévy processes · Gamma processes - Gamma bridges · Markov process · Bayes theorem

Mathematics Subject Classification (2010) 60G40 • 60G46 · 60G51 · 60H10 • 60J75

Astrid Hilbert

astrid.hilbert@lnu.se

Mohamed Erraoui

erraoui@uca.ac.ma

Mohammed Louriki

louriki.1992@gmail.com

1 Département de Mathématiques, Faculté des Sciences Semlalia, Université Cadi Ayyad, B.P. 2390, Marrakech, Maroc

2 School of Computer Science, Physics and Mathematics, Linnaeus University, Vejdesplats 7, 35195 Växjö, Sweden 


\section{Introduction}

The gamma process has proven very successful when modelling accumulation processes. Early studies by Hammersley [14], Moran [21], Gani [13], Kendall [18], Kingman [19] addressed the modelling of water stored in and released from reservoirs and accumulation related to storage in general. Dufresne et al. [9] show how to employ the gamma process to model liabilities of insurance portfolios for continuous claims. For these risk models, the fixed budget horizon of one year is assumed. The authors investigate furthermore the gamma process in the setting of ruin theory and supply ruin probabilities in form of tables. The gamma process replaces the compound processes used traditionally. In Emery and Yor [12] and Yor [25], gamma bridges were studied and their application to stop loss reinsurance and credit risk management was pointed out in Brody et al. [8]. This work introduces and focusses on random gamma bridges, which model accumulated losses of large credit portfolios in credit risk management. These studies were continued by Hoyle et al. in a series of papers, see, e.g. Hoyle and Mengütürk [16]. In a further article, pricing at an intermediate time is studied Hoyle et al. [15]. Returning to the starting point accumulation processes for storage, we refer to recent developments in Chan et al. [10] for further references. Numerical results may be found in Assmusen and Hobolth [2].

In this paper, we generalize the concept of a gamma bridge to random times, at which the bridge is pinned, to study amongst others its Markov property and to give its decomposition semi-martingale. There are two recent works in which bridges with random length are studied. The first by Bedini et al. [5] studies related properties of the Brownian bridge with random length, and the second by Erraoui and Louriki [11] studies Gaussian bridges with random length. In both works, existence of an explicit expression for the bridge with random length is exploited. Applications for the gamma bridge with random length suggest itself for accumulation processes in financial mathematics, see [3].

The paper is organized as follows. Section 2 begins by recalling the definitions and some properties of gamma processes and gamma bridges of deterministic length, which will be used throughout the paper. In Sect. 3, we define the gamma bridge with random time $\tau$ which will be denoted by $\zeta$ and we consider the stopping time property of $\tau$ with respect to the right continuous and completed filtration $\mathbb{F}_{+}^{\zeta, c}$ generated by the process $\zeta$. Moreover, we give the conditional distribution of $\tau$ and $\zeta_{u}$ given $\zeta_{t}$ for $u>t>0$. Next, we establish the Markov property of the process $\zeta$ with respect to its completed natural filtration. As a consequence, we derive Bayesian estimates for the distribution of the default time $\tau$, given the past behaviour of the process $\zeta$ up to time $t$. After that, we study the Markov property of the gamma bridge with random length, with respect to $\mathbb{F}_{+}^{\zeta, c}$. Finally, we give its semi-martingale decomposition with respect to $\mathbb{F}_{+}^{\zeta, c}$.

The following notation will be used throughout the paper: for a complete probability space $(\Omega, \mathscr{F}, \mathbb{P}), \mathscr{N}_{p}$ denotes the collection of $\mathbb{P}$-null sets. If $\theta$ is a random variable, then $\mathbb{P}_{\theta}$ denotes the law of $\theta$ under $\mathbb{P} . \mathscr{D}$ denotes the space of right-continuous functions with left limits (càdlàg) from $\mathbb{R}_{+}$to $\mathbb{R}_{+}$, endowed with Skorohod's topology, under which the space $\mathscr{D}$ is a Polish space. If $E$ is a topological space, then the Borel $\sigma$ algebra over $E$ will be denoted by $\mathscr{B}(E)$. The characteristic function of a set $A$ is 
written $\mathbb{I}_{A}$. The symmetric difference of two sets $A$ and $B$ is denoted by $A \Delta B$. Finally for any process $Y=\left(Y_{t}, t \geq 0\right)$ on $(\Omega, \mathscr{F}, \mathbb{P})$, we define by:

(i) $\mathbb{F}^{Y}=\left(\mathscr{F}_{t}^{Y}:=\sigma\left(Y_{s}, s \leq t\right), t \geq 0\right)$ the natural filtration of the process $Y$.

(ii) $\mathbb{F}^{Y, c}=\left(\mathscr{F}_{t}^{Y, c}:=\mathscr{F}_{t}^{Y} \vee \mathscr{N}_{P}, t \geq 0\right)$ the completed natural filtration of the process $Y$.

(iii) $\mathbb{F}_{+}^{Y, c}=\left(\mathscr{F}_{t+}^{Y, c}:=\bigcap_{s>t} \mathscr{F}_{s}^{Y, c}=\mathscr{F}_{t^{+}}^{Y} \vee \mathscr{N}_{P}, t \geq 0\right)$ the smallest filtration containing $\mathbb{F}^{Y}$ and satisfying the usual hypotheses of right-continuity and completeness.

\section{Gamma and Gamma Bridge Processes}

The purpose of this section is to recall the definition and some properties of the standard gamma process and the gamma bridge with deterministic length.

\subsection{Gamma Process}

By a standard gamma process $\left(\gamma_{t}, t \geq 0\right)$ on $(\Omega, \mathscr{F}, \mathbb{P})$, we mean a subordinator without drift having the Lévy-Khintchine representation given by

$$
\begin{aligned}
\mathbb{E}\left(\exp \left(-\lambda \gamma_{t}\right)\right) & =\exp \left(-t \int_{0}^{\infty}(1-\exp (-\lambda x)) \frac{\exp (-x)}{x} \mathrm{~d} x\right) \\
& =(1+\lambda)^{-t}
\end{aligned}
$$

where $v(\mathrm{~d} x)=\frac{\exp (-x)}{x} \mathbb{I}_{(0, \infty)}(x) \mathrm{d} x$ is the so-called Lévy measure. We note that the formula (2) is obtained from (1) using the Frullani formula.

The following properties, inferred from (2) by means of standard arguments (see Theorems 21.1-21.9, pp. 135-140 in Chapter 4 of Sato [22]), describe the paths of the gamma process.

Proposition 1 The gamma process $\left(\gamma_{t}, t \geq 0\right)$ has the following properties:

(i) $\gamma$ is a purely jump process;

(ii) $\gamma$ is not a compound Poisson process, and its jumping times are countable and dense in $[0, \infty)$ a.s.;

(iii) the map $t \mapsto \gamma_{t}$ is strictly increasing and not continuous anywhere a.s.;

(iv) $\gamma$ has sample paths of finite variation a.s.;

(v) $\gamma_{t}, t>0$, follows a gamma distribution with density

$$
f_{\gamma_{t}}(x)=\frac{x^{t-1} \exp (-x)}{\Gamma(t)} \mathbb{I}_{(0, \infty)}(x),
$$

where $\Gamma$ is the gamma function. 
The second property means that, for any $t>0, \gamma$ has infinite activity, that is, almost all paths have infinitely many jumps along any time interval of finite length. It is a direct consequence of $v\left(\mathbb{R}_{+}\right)=+\infty$, whereas the fourth property arises from $\int_{0}^{1} x v(\mathrm{~d} x)<+\infty$.

Remark 1 1. It is clear that the process gamma $\left(\gamma_{t}, t \geq 0\right)$ is a process with paths in $\mathscr{D}$.

2. The process $\left(\gamma_{t}-\gamma_{t^{-}}:=e_{t}, t \geq 0\right)$ of jumps of the gamma process $\left(\gamma_{t}, t \geq 0\right)$ is a Poisson point process whose intensity measure is the Lévy measure of $\left(\gamma_{t}, t \geq 0\right)$, see Theorem 1, p. 13 of Bertoin [4]. For $r>0$, let us denote by $\left(J_{1}^{r} \geq J_{2}^{r} \geq \cdots\right)$ the sequel of the lengths of jumps of the process $\left(\gamma_{t}, t \in[0, r]\right)$ ranked in decreasing order. It is not difficult to see that since the intensity measure of the Poisson point process $\left.\left(\left(t, e_{t}\right), t \geq 0\right)\right)$ is $\mathrm{d} t \frac{\exp (-x)}{x} \mathbb{I}_{(0, \infty)}(x) \mathrm{d} x$, the jump times $\left(U_{1}^{r}, U_{2}^{r}, \ldots\right)$ constitute a sequence of i.i.d r.v.'s with uniform law on $[0, r]$ which is independent from the sequence $\left(J_{k}^{r}, k \geq 1\right)$. Thus, we have the following representation:

$$
\gamma_{t}=\sum_{k \geq 1} J_{k}^{r} \mathbb{I}_{\left\{U_{k}^{r} \leq t\right\}}, \quad t \in[0, r] .
$$

We note that: $\gamma_{r}=\sum_{k \geq 1} J_{k}^{r}$.

The next proposition gives three other useful properties of the gamma process.

Proposition 2 (i) For every $r>0$, the $\sigma$-algebras, $\sigma\left(\frac{\gamma_{u}}{\gamma_{r}}, u \in[0, r]\right)$ and $\sigma\left(\gamma_{u}, u \in\right.$ $[r, \infty))$ are independent.

(ii) For any $r>0,\left(\gamma_{t}, 0 \leq t \leq r\right)$ satisfies the following equation

$$
\gamma_{t}=M_{t}^{r}+\int_{0}^{t} \frac{\gamma_{r}-\gamma_{s}}{r-s} d s
$$

where $\left(M_{t}^{r}, t \in[0, r]\right)$ is a $\mathscr{G}_{t}^{(r)}$-martingale with $\mathscr{G}_{t}^{(r)}=\sigma\left(\gamma_{s}, s \in[0, t] \cup\{r\}\right)$. (iii) $\left(\gamma_{t}, t \geq 0\right)$ has the Markov property with respect to its natural filtration.

Proof For (i) and (ii) See, [12]. (iii) $\left(\gamma_{t}, t \geq 0\right)$ has the Markov property since it is a Lévy process.

For a deeper investigation on the properties of the gamma process, we refer to Kyprianou [20], Sato [22] and Yor [25].

\subsection{Gamma Bridge with Deterministic Length}

A bridge is a stochastic process that is pinned to some fixed point at a fixed future time. In this section, we define the gamma bridge with deterministic length and we give some important properties of this process. For fixed $r>0$, we define the gamma bridge of length $r$ by setting 
Definition 1 Let $r \in(0,+\infty)$. The map $\zeta^{r}: \Omega \longmapsto \mathscr{D}$ defined by

$$
\zeta_{t}^{r}(\omega):=\frac{\gamma_{t \wedge r}(\omega)}{\gamma_{r}(\omega)}, \quad t \geq 0, \omega \in \Omega
$$

is the bridge associated with the standard gamma process $\left(\gamma_{t}, t \geq 0\right)$. Then, clearly $\zeta_{0}^{r}=0$ and $\zeta_{r}^{r}=1$. We refer to $\zeta^{r}$ as the standard gamma bridge of length $r$ associated with $\gamma$. We note that $\zeta^{r}$ is also called the Dirichlet process with parameter $r$.

We note that the process $\zeta^{r}$ is really a function of the variables $(r, t, \omega)$, and for technical reasons, it is convenient to have certain joint measurability properties.

Lemma 1 The map $(r, t, \omega) \longmapsto \zeta_{t}^{r}(\omega)$ of $\left((0,+\infty) \times \mathbb{R}_{+} \times \Omega, \mathscr{B}((0,+\infty)) \otimes\right.$ $\left.\mathscr{B}\left(\mathbb{R}_{+}\right) \otimes \mathscr{F}\right)$ into $\left(\mathbb{R}_{+}, \mathscr{B}\left(\mathbb{R}_{+}\right)\right)$is measurable. In particular, the t-section of $(r, t, \omega) \longmapsto \zeta_{t}^{r}(\omega):(r, \omega) \longmapsto \zeta_{t}^{r}(\omega)$ is measurable with respect to the $\sigma$-algebra $\mathscr{B}((0,+\infty)) \otimes \mathscr{F}$, for all $t \geq 0$.

Proof Since the map $(r, t) \longmapsto t \wedge r$ is Lipschitz continuous and $t \mapsto \gamma_{t}$ is càdlàg for almost all $\omega \in \Omega$, the map $(r, t, \omega) \longmapsto \zeta_{t}^{r}(\omega)$ can be obtained as the pointwise limit of sequences of measurable functions. So, it is sufficient to use standard results on the passage to the limit of sequences of measurable functions.

As a consequence, we have the following corollary.

Corollary 1 The map $(r, \omega) \longmapsto \zeta_{t}^{r}(\omega)$ of $((0,+\infty) \times \Omega, \mathscr{B}((0,+\infty)) \otimes \mathscr{F})$ into $(\mathscr{D}, \mathscr{B}(\mathscr{D}))$ is measurable.

A number of properties of the gamma bridge $\zeta^{r}$ sample paths can be easily deduced from the corresponding properties of the gamma sample paths. Hence, we have

Proposition 3 The gamma bridge $\zeta_{t}^{r}, t \geq 0$, has the following properties:

(i) $\zeta^{r}$ is a purely jump process, and its jumping times are countable and dense in $[0, r]$ a.s.;

(ii) the map $t \mapsto \zeta_{t}^{r}$ is strictly increasing and not continuous anywhere in $[0, r]$ a.s.;

(iii) $\zeta^{r}$ has sample paths of finite variation in $[0,+\infty)$ a.s.;

(iv) $\zeta^{r}$ has the following representation:

$$
\zeta_{t}^{r}=\sum_{k \geq 1} \frac{J_{k}^{r}}{\sum_{j \geq 1} J_{j}^{r}} \mathbb{I}_{\left\{U_{k}^{r} \leq t\right\}}, \quad t \geq 0 .
$$

We now turn to distributional properties of the gamma bridge.

Proposition 4 (i) For all $0<t<r$, the random variable $\zeta_{t}^{r}$ has a beta distribution $\beta(t, r-t)$, i.e. its density function is given by

$$
\varphi_{\zeta_{t}^{r}}(x)=\frac{\Gamma(r)}{\Gamma(t) \Gamma(r-t)} x^{t-1}(1-x)^{r-t-1} \mathbb{I}_{(0,1)}(x) .
$$


(ii) For any $0=t_{0}<t_{1}<\cdots<t_{n}=r$, the vector $\left(\zeta_{t_{1}}^{r}-\zeta_{t_{0}}^{r}, \ldots, \zeta_{t_{n}}^{r}-\zeta_{t_{n-1}}^{r}\right)$ is independent from $\gamma_{r}$, with density

$$
\frac{\Gamma(r)}{\prod_{i=1}^{n} \Gamma\left(t_{i}-t_{i-1}\right)} \prod_{i=1}^{n} x_{i}^{t_{i}-t_{i-1}-1}
$$

with respect to the Lebesgue measure $d x_{1} \ldots d x_{n-1}$ (or, as well, $\left.d x_{2} \ldots d x_{n}\right)$ on the simplex

$$
\left\{\left(x_{1}, \ldots, x_{n}\right): x_{i} \geq 0, x_{1}+\cdots+x_{n}=1\right\} .
$$

(iii) For all $t<u<r$ and $x \in(0,1)$, the regular conditional law of $\zeta_{u}^{r}$ given $\zeta_{t}^{r}=x$ is given by:

$$
\begin{aligned}
& \mathbb{P}\left(\zeta_{u}^{r} \in d y \mid \zeta_{t}^{r}=x\right) \\
& \quad=\frac{\Gamma(r-t)}{\Gamma(u-t) \Gamma(r-u)} \frac{(y-x)^{u-t-1}(1-y)^{r-u-1}}{(1-x)^{r-t-1}} \mathbb{I}_{\{x<y<1\}} d y .
\end{aligned}
$$

In the same spirit as in Proposition 2, we have

Proposition 5 (i) $\zeta^{r}$ is a Markov process with respect to its natural filtration.

(ii) $\zeta^{r}$ satisfies the following equation

$$
\zeta_{t}^{r}=N_{t}^{r}+\int_{0}^{t} \frac{1-\zeta_{s}^{r}}{r-s} d s, \quad t \in[0, r]
$$

where $\left(N_{t}^{r}, t \in[0, r]\right)$ is a $\mathbb{F}^{r}$-martingale.

Proof (i) From Theorem 1.3 in Blumenthal and Getoor [6], it suffices to prove that for every bounded measurable function $g$ we have:

$$
\mathbb{E}\left[g\left(\zeta_{u}^{r}\right) \mid \zeta_{t_{1}}^{r}, \ldots, \zeta_{t_{n}}^{r}\right]=\mathbb{E}\left[g\left(\zeta_{u}^{r}\right) \mid \zeta_{t_{n}}^{r}\right]
$$

for all $0 \leq t_{1}<\ldots<t_{n}<u \leq r$ and for all $n \geq 1$.

Using Proposition 2 (i), we have

$$
\begin{aligned}
\mathbb{E}\left[g\left(\zeta_{u}^{r}\right) \mid \zeta_{t_{1}}^{r}, \ldots, \zeta_{t_{n}}^{r}\right] & =\mathbb{E}\left[g\left(\frac{\gamma_{u}}{\gamma_{r}}\right) \mid \frac{\gamma_{t_{1}}}{\gamma_{r}}, \ldots, \frac{\gamma_{t_{n}}}{\gamma_{r}}\right] \\
& =\mathbb{E}\left[g\left(\frac{\gamma_{u}}{\gamma_{r}}\right) \mid \frac{\gamma_{t_{1}}}{\gamma_{t_{2}}}, \frac{\gamma_{t_{2}}}{\gamma_{t_{3}}}, \ldots, \frac{\gamma_{t_{n-1}}}{\gamma_{t_{n}}}, \frac{\gamma_{t_{n}}}{\gamma_{r}}\right] \\
& =\mathbb{E}\left[g\left(\frac{\gamma_{u}}{\gamma_{r}}\right) \mid \frac{\gamma_{t_{n}}}{\gamma_{r}}\right] \\
& =\mathbb{E}\left[g\left(\zeta_{u}^{r}\right) \mid \zeta_{t_{n}}^{r}\right] .
\end{aligned}
$$


Hence, the formula (11) is proved; then, $\zeta^{r}$ is a Markov process with respect to its natural filtration.

(ii) We have from Proposition 2 (ii) that

$$
\gamma_{t}=M_{t}^{r}+\int_{0}^{t} \frac{\gamma_{r}-\gamma_{s}}{r-s} \mathrm{~d} s, \quad t \in[0, r]
$$

where $M^{r}$ is a martingale with respect to the filtration $\mathscr{G}_{t}^{(r)}=\sigma\left(\gamma_{s}, s \in[0, t] \cup\{r\}\right)$. Then, it is easy to see that

$$
\zeta_{t}^{r}=N_{t}^{r}+\int_{0}^{t} \frac{1-\zeta_{s}^{r}}{r-s} \mathrm{~d} s, \quad t \in[0, r]
$$

where $N_{t}^{r}=\frac{M_{t}^{r}}{\gamma_{r}}, t \in[0, r]$. Firstly, notice that $\mathscr{F}_{t}^{\zeta^{r}} \subset \mathscr{G}_{t}^{(r)}$ and $\gamma_{r}$ is $\mathscr{G}_{t}^{(r)}$-measurable for all $t \leq r$. Moreover, Eq. (12) yields, that is the process $N^{r}$ is $\mathbb{F}^{\zeta^{r}}$-adapted. In view of these considerations, as well as the fact that $M_{t}^{r}$ is a $\mathscr{G}_{t}^{(r)}$-martingale we obtain

$$
\begin{aligned}
\mathbb{E}\left[N_{t}^{r} \mid \mathscr{F}_{s}^{\zeta^{r}}\right] & =\mathbb{E}\left[\frac{M_{t}^{r}}{\gamma_{r}} \mid \mathscr{F}_{s}^{\zeta^{r}}\right]=\mathbb{E}\left[\mathbb{E}\left[\frac{M_{t}^{r}}{\gamma_{r}} \mid \mathscr{G}_{s}^{(r)}\right] \mid \mathscr{F}_{s}^{\zeta^{r}}\right] \\
& =\mathbb{E}\left[\frac{M_{s}^{r}}{\gamma_{r}} \mid \mathscr{F}_{s}^{\zeta^{r}}\right]=\mathbb{E}\left[N_{s}^{r} \mid \mathscr{F}_{s}^{\zeta^{r}}\right]=N_{s}^{r},
\end{aligned}
$$

for $0 \leq s \leq t \leq r$. It follows that $\left(N_{t}^{r}, t \in[0, r]\right)$ is a $\mathbb{F}^{\zeta^{r}}$-martingale. Hence, equation (10) is satisfied.

Remark 2 We can rewrite (5) in the form

$$
\gamma_{t \wedge r}=M_{t \wedge r}^{r}+\int_{0}^{t \wedge r} \frac{\gamma_{r}-\gamma_{s}}{r-s} \mathrm{~d} s, \quad t \geq 0 .
$$

Then, we obtain

$$
\zeta_{t}^{r}=\frac{\gamma_{t \wedge r}}{\gamma_{r}}=\frac{M_{t \wedge r}^{r}}{\gamma_{r}}+\int_{0}^{t \wedge r} \frac{1-\zeta_{s}^{r}}{r-s} \mathrm{~d} s, \quad t \geq 0
$$

For every $t \geq 0$, we set $\widehat{N}_{t}^{r}=\frac{M_{t \wedge r}^{r}}{\gamma_{r}}$. We have thus

$$
\zeta_{t}^{r}=\widehat{N}_{t}^{r}+\int_{0}^{t} \frac{1-\zeta_{s}^{r}}{r-s} \mathbb{I}_{\{s<r\}} \mathrm{d} s, \quad t \geq 0 .
$$

It follows from the above proposition that $\left(\widehat{N}_{t}^{r}, t \geq 0\right)$ is a $\mathbb{F}^{\zeta}$-martingale stopped at $r$. 


\section{Gamma Bridges with Random Length}

In this section, we define and study a process $\left(\zeta_{t}, t \geq 0\right)$ which generalizes the gamma bridge in the sense that the time $r$ at which the bridge is pinned is substituted by an independent random time $\tau$. We call it gamma bridge with random length. We prove that the random time $\tau$ is a stopping time with respect to the completed filtration $\mathbb{F}^{\zeta, c}$, and we give the regular conditional distribution of $\tau$ and $(\tau, \zeta$.) given $\zeta$. Moreover, we prove that the gamma bridge with random length $\zeta$ is an in-homogeneous Markov process with respect to its completed natural filtration $\mathbb{F}^{\zeta}, c$ as well as with respect to $\mathbb{F}_{+}^{\zeta, c}$. The last property allows us to deduce an interesting consequence, that is the filtration $\mathbb{F}^{\zeta, c}$ satisfies the usual conditions of completeness and right-continuity. Finally, we give the semi-martingale decomposition of $\zeta$ with respect to $\mathbb{F}_{+}^{\zeta, c}$.

Now, we give precise definition of the process $\left(\zeta_{t}, t \geq 0\right)$. Due to Corollary 1 we could substitute $r$ by a random time $\tau$ in (6). Thus, we obtain

Definition 2 Let $\tau:(\Omega, \mathscr{F}, \mathbb{P}) \longmapsto(0,+\infty)$ be a strictly positive random time, with distribution function $F(t):=\mathbb{P}(\tau \leq t), t \geq 0$. The map $\zeta:(\Omega, \mathscr{F}) \longrightarrow(\mathscr{D}, \mathscr{B}(\mathscr{D}))$ is defined by

$$
\zeta_{t}(\omega):=\left.\zeta_{t}^{r}(\omega)\right|_{r=\tau(\omega)}, \quad(t, \omega) \in \mathbb{R}_{+} \times \Omega
$$

Then, $\zeta$ takes the form

$$
\zeta_{t}:=\frac{\gamma_{t \wedge \tau}}{\gamma_{\tau}}, \quad t \geq 0
$$

Since $\zeta$ is obtained by composition of two maps $(r, t, \omega) \longmapsto \zeta_{t}^{r}(\omega)$ and $(t, \omega) \longmapsto$ $(\tau(\omega), t, \omega)$, it is not hard to verify that the map $\zeta:(\Omega, \mathscr{F}) \longrightarrow(\mathscr{D}, \mathscr{B}(\mathscr{D}))$ is measurable. The process $\zeta$ will be called gamma bridge with random length $\tau$.

As mentioned above, we work under the following standing assumption:

Assumption 1 The random time $\tau$ and the gamma process $\gamma$ are independent.

Using the fact that the process $\zeta$ is obtained by the substitution of $r$ in $\zeta^{r}$ by the random time $\tau$ allows us to derive a lot of information about its path properties. Hence, we have

Proposition 6 The gamma bridge $\left(\zeta_{t}, t \geq 0\right)$ with random length $\tau$ has the following properties:

(i) $\zeta$ is a purely jump process, and its jumping times are countable and dense in $[0, \tau]$ a.s.;

(ii) the map $t \mapsto \zeta_{t}$ is increasing and not continuous anywhere on $[0, \tau]$ a.s.;

(iii) $\zeta$ has sample paths of finite variation a.s.

(iv) $\zeta$ has the following representation:

$$
\zeta_{t}=\sum_{k \geq 1} \frac{J_{k}^{\tau}}{\sum_{j \geq 1} J_{j}^{\tau}} \mathbb{I}_{\left\{U_{k}^{\tau} \leq t\right\}}, \quad t \geq 0
$$


where the jump times $\left(U_{1}^{\tau}, U_{2}^{\tau}, \ldots\right)$ constitute a sequence of r.v.'s identically distributed with the law given by

$$
\begin{aligned}
\mathbb{P}\left[U_{k}^{\tau} \leq t\right] & =\mathbb{P}[\tau \leq t]+\int_{(t,+\infty)} \mathbb{P}\left[U_{k}^{r} \leq t\right] \mathbb{P}_{\tau}(d r) \\
& =\mathbb{P}[\tau \leq t]+t \mathbb{E}\left[\frac{1}{\tau} \mathbb{I}_{(\tau>t)}\right], \quad t \geq 0, k \geq 1 .
\end{aligned}
$$

\subsection{Stopping Time Property of $\tau$}

The aim of this subsection is to prove that the random time $\tau$ is a stopping time with respect to $\mathbb{F}^{\zeta, c}$.

Proposition 7 Forall $t>0$, we have $\mathbb{P}\left(\left\{\zeta_{t}=1\right\} \triangle\{\tau \leq t\}\right)=0$. Then, $\tau$ is a stopping time with respect to $\mathbb{F}^{\zeta, c}$ and consequently it is a stopping time with respect to $\mathbb{F}_{+}^{\zeta, c}$.

Proof First, we have from the definition of $\zeta$ that $\zeta_{t}=1$ for $\tau \leq t$. Then, $\{\tau \leq t\} \subseteq$ $\left\{\zeta_{t}=1\right\}$. On the other hand, using the formula of total probability we obtain

$$
\begin{aligned}
\mathbb{P}\left(\zeta_{t}=1, t<\tau\right) & =\int_{(t,+\infty)} \mathbb{P}\left(\zeta_{t}=1 \mid \tau=r\right) \mathbb{P}_{\tau}(\mathrm{d} r) \\
& =\int_{(t,+\infty)} \mathbb{P}\left(\zeta_{t}^{r}=1\right) \mathbb{P}_{\tau}(\mathrm{d} r) \\
& =0 .
\end{aligned}
$$

The latter equality uses the fact that $\zeta_{t}^{r}$ is a random variable having a beta distribution for $0<t<r$. Thus, $\mathbb{P}\left(\left\{\zeta_{t}=1\right\} \triangle\{\tau \leq t\}\right)=0$. It follows that the event $\{\tau \leq t\}$ belongs to $\mathscr{F}_{t}^{\zeta} \vee \mathscr{N}_{P}$, for all $t \geq 0$. Hence, $\tau$ is a stopping time with respect to $\mathbb{F}^{\zeta, c}$ and consequently it is also a stopping time with respect to $\mathbb{F}_{+}^{\zeta, c}$.

In order to determine the conditional law of the random time $\tau$ given $\zeta_{t}$, we will use the following

Proposition 8 Let $t>0$ such that $F(t)>0$. Let $g: \mathbb{R}_{+} \longrightarrow \mathbb{R}$ be a Borel function satisfying $\mathbb{E}[|g(\tau)|]<+\infty$. Then, $\mathbb{P}$-a.s., we have

$$
\mathbb{E}\left[g(\tau) \mid \zeta_{t}\right]=\int_{(0, t]} \frac{g(r)}{F(t)} \mathbb{P}_{\tau}(d r) \mathbb{I}_{\left\{\zeta_{t}=1\right\}}+\int_{(t,+\infty)} g(r) \phi_{\zeta_{t}^{r}}\left(\zeta_{t}\right) \mathbb{P}_{\tau}(d r) \mathbb{I}_{\left\{0<\zeta_{t}<1\right\}},
$$


where the function $\phi_{\zeta_{t}^{r}}$ is defined on $\mathbb{R}$ by:

$$
\begin{aligned}
\phi_{\zeta_{t}^{r}}(x) & =\frac{\varphi_{\zeta_{t}^{r}}(x)}{\int_{(t,+\infty)} \varphi_{\zeta_{t}^{s}}(x) \mathbb{P}_{\tau}(d s)} \\
& =\frac{(1-x)^{r} \frac{\Gamma(r)}{\Gamma(r-t)}}{\int_{(t,+\infty)}(1-x)^{s} \frac{\Gamma(s)}{\Gamma(s-t)} \mathbb{P}_{\tau}(d s)} \mathbb{I}_{(0,1)}(x), \quad x \in \mathbb{R}, \quad r \in(t,+\infty) .
\end{aligned}
$$

Proof Let us consider the measure $\mu$ defined on $\mathscr{B}(\mathbb{R})$ by

$$
\mu(\mathrm{d} x)=\delta_{1}(\mathrm{~d} x)+\mathrm{d} x
$$

where $\delta_{1}(\mathrm{~d} x)$ and $\mathrm{d} x$ are the Dirac measure and the Lebesgue measure on $\mathscr{B}(\mathbb{R})$, respectively. Then, for any $B \in \mathscr{B}(\mathbb{R})$ we have

$$
\mathbb{P}\left(\zeta_{t} \in B \mid \tau=r\right)=\mathbb{P}\left(\zeta_{t}^{r} \in B\right)=\int_{B} q_{t}(r, x) \mu(\mathrm{d} x)
$$

where the function $q_{t}$ is non-negative and measurable in the two variables jointly given by

$$
q_{t}(r, x)=\mathbb{I}_{\{x=1\}} \mathbb{I}_{\{r \leq t\}}+\varphi_{\zeta_{t}^{r}}(x) \mathbb{I}_{\{0<x<1\}} \mathbb{I}_{\{t<r\}}
$$

It follows from Bayes formula (see [23], p. 272) that $\mathbb{P}$-a.s.:

$$
\begin{aligned}
\mathbb{E}\left[g(\tau) \mid \zeta_{t}\right] & =\frac{\int_{(0,+\infty)} g(r) q_{t}\left(r, \zeta_{t}\right) \mathbb{P}_{\tau}(\mathrm{d} r)}{\int_{(0,+\infty)} q_{t}\left(r, \zeta_{t}\right) \mathbb{P}_{\tau}(\mathrm{d} r)} \\
& =\frac{\int_{(0, t]} g(r) \mathbb{P}_{\tau}(\mathrm{d} r) \mathbb{I}_{\left\{\zeta_{t}=1\right\}}+\int_{(t,+\infty)} g(r) \varphi_{\zeta_{t}^{r}}\left(\zeta_{t}\right) \mathbb{P}_{\tau}(\mathrm{d} r) \mathbb{I}_{\left\{0<\zeta_{t}<1\right\}}}{F(t) \mathbb{I}_{\left\{\zeta_{t}=1\right\}}+\int_{(t,+\infty)} \varphi_{\zeta_{t}^{r}}\left(\zeta_{t}\right) \mathbb{P}_{\tau}(\mathrm{d} r) \mathbb{I}_{\left\{0<\zeta_{t}<1\right\}}} \\
& =\int_{(0, t]} \frac{g(r)}{F(t)} \mathbb{P}_{\tau}(\mathrm{d} r) \mathbb{I}_{\left\{\zeta_{t}=1\right\}}+\int_{(t,+\infty)} g(r) \phi_{\zeta_{t}^{r}}\left(\zeta_{t}\right) \mathbb{P}_{\tau}(\mathrm{d} r) \mathbb{I}_{\left\{0<\zeta_{t}<1\right\}} .
\end{aligned}
$$


Corollary 2 The conditional law of the random time $\tau$ given $\zeta_{t}$ is given by

$$
\mathbb{P}_{\tau \mid \zeta_{t}=x}(x, d r)=\frac{1}{F(t)} \mathbb{I}_{\{x=1\}} \mathbb{I}_{(0, t]}(r) \mathbb{P}_{\tau}(d r)+\phi_{\zeta_{t}^{r}}(x) \mathbb{I}_{\{0<x<1\}} \mathbb{I}_{(t,+\infty)}(r) \mathbb{P}_{\tau}(d r)
$$

The previous proposition can be expanded as follows:

Proposition 9 Let $u>t>0$ such that $F(t)>0$. Let $\mathfrak{g}$ be a bounded measurable function defined on $(0,+\infty) \times \mathbb{R}$. Then, $\mathbb{P}$-a.s., we have

$$
\begin{aligned}
\mathbb{E}\left[\mathfrak{g}\left(\tau, \zeta_{t}\right) \mid \zeta_{t}\right]= & \int_{(0, t]} \frac{\mathfrak{g}(r, 1)}{F(t)} \mathbb{P}_{\tau}(d r) \mathbb{I}_{\left\{\zeta_{t}=1\right\}} \\
& +\int_{(t,+\infty)} \mathfrak{g}\left(r, \zeta_{t}\right) \phi_{\zeta_{t}}\left(\zeta_{t}\right) \mathbb{P}_{\tau}(d r) \mathbb{I}_{\left\{0<\zeta_{t}<1\right\}},
\end{aligned}
$$

and

$$
\begin{aligned}
\mathbb{E}\left[\mathfrak{g}\left(\tau, \zeta_{u}\right) \mid \zeta_{t}\right]= & \int_{(0, t]} \frac{\mathfrak{g}(r, 1)}{F(t)} \mathbb{P}_{\tau}(d r) \mathbb{I}_{\left\{\zeta_{t}=1\right\}}+\int_{(t, u]} \mathfrak{g}(r, 1) \phi_{\zeta_{t}^{r}}\left(\zeta_{t}\right) \mathbb{P}_{\tau}(d r) \mathbb{I}_{\left\{0<\zeta_{t}<1\right\}} \\
& +\int_{(u,+\infty)} \mathfrak{G}_{t, u}\left(r, \zeta_{t}\right) \phi_{\zeta_{t}^{r}}\left(\zeta_{t}\right) \mathbb{P}_{\tau}(d r) \mathbb{I}_{\left\{0<\zeta_{t}<1\right\}}
\end{aligned}
$$

Here, the function $\mathfrak{G}_{t, u}(r, \cdot)$ is defined by

$$
\begin{aligned}
\mathfrak{G}_{t, u}(r, x) & :=\mathbb{E}\left[\mathfrak{g}\left(r, \zeta_{u}^{r}\right) \mid \zeta_{t}^{r}=x\right] \\
& =\int_{\mathbb{R}} \mathfrak{g}(r, y) \mathbb{P}_{\zeta_{u}^{r} \mid \zeta_{t}^{r}=x}(d y) .
\end{aligned}
$$

Proof First of all, it is easy to see that (20) is an immediate consequence of Proposition 8. Now, to show (21) we begin with by splitting $\mathbb{E}\left[\mathfrak{g}\left(\tau, \zeta_{u}\right) \mid \zeta_{t}\right]$ as follows:

$\mathbb{E}\left[\mathfrak{g}\left(\tau, \zeta_{u}\right) \mid \zeta_{t}\right]=\mathbb{E}\left[\mathfrak{g}(\tau, 1) \mathbb{I}_{\{\tau \leq t\}} \mid \zeta_{t}\right]+\mathbb{E}\left[\mathfrak{g}(\tau, 1) \mathbb{I}_{\{t<\tau \leq u\}} \mid \zeta_{t}\right]+\mathbb{E}\left[\mathfrak{g}\left(\tau, \zeta_{u}\right) \mathbb{I}_{\{u<\tau\}} \mid \zeta_{t}\right]$

We obtain from Proposition 8 that

$$
\mathbb{E}\left[\mathfrak{g}(\tau, 1) \mathbb{I}_{\{\tau \leq t\}} \mid \zeta_{t}\right]=\int_{(0, t]} \frac{\mathfrak{g}(r, 1)}{F(t)} \mathbb{P}_{\tau}(\mathrm{d} r) \mathbb{I}_{\left\{\zeta_{t}=1\right\}}
$$

and

$$
\mathbb{E}\left[\mathfrak{g}(\tau, 1) \mathbb{I}_{\{t<\tau \leq u\}} \mid \zeta_{t}\right]=\int_{(t, u]} \mathfrak{g}(r, 1) \phi_{\zeta_{t}^{r}}\left(\zeta_{t}\right) \mathbb{P}_{\tau}(\mathrm{d} r) \mathbb{I}_{\left\{0<\zeta_{t}<1\right\}}
$$


Next, we prove that

$$
\mathbb{E}\left[\mathfrak{g}\left(\tau, \zeta_{u}\right) \mathbb{I}_{\{u<\tau\}} \mid \zeta_{t}\right]=\int_{(u,+\infty)} \mathfrak{G}_{t, u}\left(r, \zeta_{t}\right) \phi_{\zeta_{t}^{r}}\left(\zeta_{t}\right) \mathbb{P}_{\tau}(\mathrm{d} r) \mathbb{I}_{\left\{0<\zeta_{t}<1\right\}}
$$

Indeed, for a bounded Borel function $h$ we have

$$
\begin{aligned}
\mathbb{E}\left[\mathfrak{g}\left(\tau, \zeta_{u}\right) \mathbb{I}_{\{u<\tau\}} h\left(\zeta_{t}\right)\right] & =\int_{(u,+\infty)} E\left[\mathfrak{g}\left(r, \zeta_{u}^{r}\right) h\left(\zeta_{t}^{r}\right)\right] \mathbb{P}_{\tau}(\mathrm{d} r) \\
& =\int_{(u,+\infty)} \mathbb{E}\left[\mathbb{E}\left[\mathfrak{g}\left(r, \zeta_{u}^{r}\right) h\left(\zeta_{t}^{r}\right) \mid \zeta_{t}^{r}\right]\right] \mathbb{P}_{\tau}(\mathrm{d} r) \\
& =\int_{(u,+\infty)} \mathbb{E}\left[\mathbb{E}\left[\mathfrak{g}\left(r, \zeta_{u}^{r}\right) \mid \zeta_{t}^{r}\right] h\left(\zeta_{t}^{r}\right)\right] \mathbb{P}_{\tau}(\mathrm{d} r)
\end{aligned}
$$

Using (22), for $t<u<r$, we get

$$
\begin{aligned}
\mathbb{E}\left[\mathfrak{g}\left(\tau, \zeta_{u}\right) \mathbb{I}_{\{u<\tau\}} h\left(\zeta_{t}\right)\right] & =\int_{(u,+\infty)} \mathbb{E}\left[\mathfrak{G}_{t, u}\left(r, \zeta_{t}^{r}\right) h\left(\zeta_{t}^{r}\right)\right] \mathbb{P}_{\tau}(\mathrm{d} r) \\
& =\mathbb{E}\left[\mathfrak{G}_{t, u}\left(\tau, \zeta_{t}\right) \mathbb{I}_{\{u<\tau\}} h\left(\zeta_{t}\right)\right]
\end{aligned}
$$

It follows from (20), that $\mathbb{P}$-a.s.

$$
\mathbb{E}\left[\mathfrak{G}_{t, u}\left(\tau, \zeta_{t}\right) \mathbb{I}_{\{u<\tau\}} \mid \zeta_{t}\right]=\int_{(u,+\infty)} \mathfrak{G}_{t, u}\left(r, \zeta_{t}\right) \phi_{\zeta_{t}^{r}}\left(\zeta_{t}\right) \mathbb{P}_{\tau}(\mathrm{d} r) \mathbb{I}_{\left\{0<\zeta_{t}<1\right\}}
$$

This induces that

$$
\mathbb{E}\left[\mathfrak{g}\left(\tau, \zeta_{u}\right) \mathbb{I}_{\{u<\tau\}} h\left(\zeta_{t}\right)\right]=\mathbb{E}\left[\int_{(u,+\infty)} \mathfrak{G}_{t, u}\left(r, \zeta_{t}\right) \phi_{\zeta_{t}^{r}}\left(\zeta_{t}\right) \mathbb{P}_{\tau}(\mathrm{d} r) \mathbb{I}_{\left\{0<\zeta_{t}<1\right\}} h\left(\zeta_{t}\right)\right]
$$

Hence, the formula (23) is proved and then the proof of the proposition is completed.

\subsection{Markov Property of $\zeta$ and Bayes Estimate of $\tau$}

In this part, we prove that the gamma bridge $\zeta$ with random length $\tau$ is an inhomogeneous Markov process with respect to its completed natural filtration $\mathbb{F}^{\zeta, c}$.

Theorem 1 The process $\left(\zeta_{t}, t \geq 0\right)$ is an $\mathbb{F}^{\zeta}$-Markov process. That is, for any $t \geq 0$, we have

$$
\mathbb{E}\left[f\left(\zeta_{t+h}\right) \mid \mathscr{F}_{t}^{\zeta}\right]=\mathbb{E}\left[f\left(\zeta_{t+h}\right) \mid \zeta_{t}\right], \mathbb{P} \text {-a.s. },
$$

for all $t, h \geq 0$ and for every bounded measurable function $f$. 
Proof First, we would like to mention that since $\zeta_{0}=0$ almost surely it is easy to see that

$$
\mathbb{E}\left[f\left(\zeta_{t+h}\right) \mid \mathscr{F}_{0}^{\zeta}\right]=\mathbb{E}\left[f\left(\zeta_{t+h}\right) \mid \zeta_{0}\right]
$$

Let us assume $t>0$. As $\mathbb{I}_{\left\{\zeta_{t}=0\right\}}=\mathbb{I}_{\{\tau \leq t\}} \mathbb{P}$-a.s, we rewrite $\mathbb{E}\left[f\left(\zeta_{t+h}\right) \mid \mathscr{F}_{t}^{\zeta}\right]$ as follows:

$$
\begin{aligned}
\mathbb{E}\left[f\left(\zeta_{t+h}\right) \mid \mathscr{F}_{t}^{\zeta}\right] & =\mathbb{E}\left[f\left(\zeta_{t+h}\right) \mid \mathscr{F}_{t}^{\zeta}\right] \mathbb{I}_{\{\tau \leq t\}}+\mathbb{E}\left[f\left(\zeta_{t+h}\right) \mid \mathscr{F}_{t}^{\zeta}\right] \mathbb{I}_{\{t<\tau\}} \\
& =f(1) \mathbb{I}_{\left\{\zeta_{t}=1\right\}}+\mathbb{E}\left[f\left(\zeta_{t+h}\right) \mid \mathscr{F}_{t}^{\zeta}\right] \mathbb{I}_{\{t<\tau\}} .
\end{aligned}
$$

So, it remains to show that

$$
\mathbb{E}\left[f\left(\zeta_{t+h}\right) \mathbb{I}_{\{t<\tau\}} \mid \mathscr{F}_{t}^{\zeta}\right]=\mathbb{E}\left[f\left(\zeta_{t+h}\right) \mathbb{I}_{\{t<\tau\}} \mid \zeta_{t}\right], \mathbb{P}-\text { a.s. }
$$

To do this, it is enough to verify that

$$
\int_{A \cap\{t<\tau\}} f\left(\zeta_{t+h}\right) d \mathbb{P}=\int_{A \cap\{t<\tau\}} \mathbb{E}\left[f\left(\zeta_{t+h}\right) \mid \zeta_{t}\right] d \mathbb{P}
$$

for all $A \in \mathscr{F}_{t}^{\zeta}$. We start by remarking that, for $t>0, \mathscr{F}_{t}^{\zeta}$ is generated by

$$
\zeta_{t_{n}}, \alpha_{n}:=\frac{\zeta_{t_{n-1}}}{\zeta_{t_{n}}}, \alpha_{n-1}=\frac{\zeta_{t_{n-2}}}{\zeta_{t_{n-1}}}, \ldots, \alpha_{2}=\frac{\zeta_{t_{1}}}{\zeta_{t_{2}}}, \alpha_{1}:=\frac{\zeta_{t_{0}}}{\zeta_{t_{1}}},
$$

$0<t_{0}<t_{1}<\cdots<t_{n}=t$ for $\mathrm{n}$ running through $\mathbb{N}$. Then, by the monotone class theorem it is sufficient to prove (26) for sets $A$ of the form $A=\left\{\zeta_{t} \in B, \alpha_{1} \in\right.$ $\left.B_{1}, \ldots, \alpha_{n} \in B_{n}\right\}$ with $B, B_{1}, B_{2}, \ldots, B_{n} \in \mathscr{B}(\mathbb{R}), n \geq 1$. Moreover, on the set $\{t<\tau\}$, we have

$$
\beta_{k}:=\frac{\gamma_{t_{k-1}}}{\gamma_{t_{k}}}=\alpha_{k}, \quad k=1, \ldots, n
$$

Using Proposition 2 (i), then for $t<r$ the vectors $\left(\beta_{1}, \ldots, \beta_{n}\right)$ and $\left(\zeta_{t}^{r}, \zeta_{t+h}^{r}\right)$ are independent. Now, taking into account all the above considerations, we have

$$
\begin{aligned}
\int_{A \cap\{t<\tau\}} f\left(\zeta_{t+h}\right) d \mathbb{P} & =\mathbb{E}\left[f\left(\zeta_{t+h}\right) \mathbb{I}_{B \times B_{1} \times \cdots \times B_{n}}\left(\zeta_{t}, \alpha_{1}, \ldots, \alpha_{n}\right) \mathbb{I}_{\{t<\tau\}}\right] \\
& =\mathbb{E}\left[f\left(\zeta_{t+h}\right) \mathbb{I}_{B \times B_{1} \times \cdots \times B_{n}}\left(\zeta_{t}, \beta_{1}, \ldots, \beta_{n}\right) \mathbb{I}_{\{t<\tau\}}\right] \\
& =\int_{(t, \infty)} \mathbb{E}\left[f\left(\zeta_{t+h}^{r}\right) \mathbb{I}_{B}\left(\zeta_{t}^{r}\right) \mathbb{I}_{B_{1} \times \cdots \times B_{n}}\left(\beta_{1}, \ldots, \beta_{n}\right)\right] \mathbb{P}_{\tau}(\mathrm{d} r) \\
& =\int_{(t, \infty)} \mathbb{E}\left[f\left(\zeta_{t+h}^{r}\right) \mathbb{I}_{B}\left(\zeta_{t}^{r}\right)\right] \mathbb{P}_{\tau}(\mathrm{d} r) \mathbb{E}\left[\mathbb{I}_{B_{1} \times \cdots \times B_{n}}\left(\beta_{1}, \ldots, \beta_{n}\right)\right] \\
& =\mathbb{E}\left[f\left(\zeta_{t+h}\right) \mathbb{I}_{B}\left(\zeta_{t}\right) \mathbb{I}_{\{t<\tau\}}\right] \mathbb{E}\left[\mathbb{I}_{B_{1} \times \cdots \times B_{n}}\left(\beta_{1}, \ldots, \beta_{n}\right)\right]
\end{aligned}
$$




$$
\begin{aligned}
& =\mathbb{E}\left[\mathbb{E}\left[f\left(\zeta_{t+h}\right) \mid \zeta_{t}\right] \mathbb{I}_{B}\left(\zeta_{t}\right) \mathbb{I}_{\{t<\tau\}}\right] \mathbb{E}\left[\mathbb{I}_{B_{1} \times \cdots \times B_{n}}\left(\beta_{1}, \ldots, \beta_{n}\right)\right] \\
& =\mathbb{E}\left[\mathbb{E}\left[f\left(\zeta_{t+h}\right) \mid \zeta_{t}\right] \mathbb{I}_{B}\left(\zeta_{t}\right) \mathbb{I}_{\{t<\tau\}} \mathbb{I}_{B_{1} \times \cdots \times B_{n}}\left(\beta_{1}, \ldots, \beta_{n}\right)\right] \\
& =\mathbb{E}\left[\mathbb{E}\left[f\left(\zeta_{t+h}\right) \mid \zeta_{t}\right] \mathbb{I}_{B}\left(\zeta_{t}\right) \mathbb{I}_{\{t<\tau\}} \mathbb{I}_{B_{1} \times \cdots \times B_{n}}\left(\alpha_{1}, \ldots, \alpha_{n}\right)\right] \\
& =\mathbb{E}\left[\mathbb{E}\left[f\left(\zeta_{t+h}\right) \mid \zeta_{t}\right] \mathbb{I}_{B \times B_{1} \times \cdots \times B_{n}}\left(\zeta_{t}, \alpha_{1}, \ldots, \alpha_{n}\right) \mathbb{I}_{\{t<\tau\}}\right] \\
& =\int_{A \cap\{t<\tau\}} \mathbb{E}\left[f\left(\zeta_{t+h}\right) \mid \zeta_{t}\right] \mathrm{d} \mathbb{P} .
\end{aligned}
$$

Hence, (26) is proved and this ends the proof.

Corollary 3 The Markov property can be extended to the completed filtration $\mathbb{F}^{\zeta, c}$.

The aim of this proposition is to provide, using the Markov property, that the observation of $\zeta_{t}$ would be sufficient to give estimates of the time $\tau$ based on the observation of the information process $\zeta$ up to time $t$.

Proposition 10 Let $0<t<u$.

(i) For each bounded measurable function g defined on $(0, \infty)$, we have $\mathbb{P}$-a.s.

$$
\mathbb{E}\left[g(\tau) \mid \mathscr{F}_{t}^{\zeta, c}\right]=g(\tau \wedge t) \mathbb{I}_{\left\{\zeta_{t}=1\right\}}+\int_{(t,+\infty)} g(r) \phi_{\zeta_{t}^{r}}\left(\zeta_{t}\right) \mathbb{P}_{\tau}(d r) \mathbb{I}_{\left\{0<\zeta_{t}<1\right\}}
$$

(ii) For each bounded measurable function defined on $(0,+\infty) \times \mathbb{R}$, we have $\mathbb{P}$-a.s.

$$
\begin{aligned}
\mathbb{E}\left[g\left(\tau, \zeta_{t}\right) \mid \mathscr{F}_{t}^{\zeta, c}\right]= & g(\tau \wedge t, 1) \mathbb{I}_{\left\{\zeta_{t}=1\right\}} \\
& +\int_{(t,+\infty)} g\left(r, \zeta_{t}\right) \phi_{\zeta_{t}^{r}}\left(\zeta_{t}\right) \mathbb{P}_{\tau}(d r) \mathbb{I}_{\left\{0<\zeta_{t}<1\right\}} \\
\mathbb{E}\left[g\left(\tau, \zeta_{u}\right) \mid \mathscr{F}_{t}^{\zeta, c}\right]= & g(\tau \wedge t, 1) \mathbb{I}_{\left\{\zeta_{t}=1\right\}} \\
& +\int_{(t, u]} g(r, 1) \phi_{\zeta_{t}^{r}}\left(\zeta_{t}\right) \mathbb{P}_{\tau}(d r) \mathbb{I}_{\left\{0<\zeta_{t}<1\right\}} \\
& +\int_{(u,+\infty)} \int_{\mathbb{R}} g(r, y) \mathbb{P}_{\zeta_{u}^{r} \mid \zeta_{t}^{r}=x}(d y) \phi_{\zeta_{t}^{r}}\left(\zeta_{t}\right) \mathbb{P}_{\tau}(d r) \mathbb{I}_{\left\{0<\zeta_{t}<1\right\}}
\end{aligned}
$$

Proof (i) Obviously, we have

$$
\mathbb{E}\left[g(\tau) \mid \mathscr{F}_{t}^{\zeta, c}\right]=\mathbb{E}\left[g(\tau \wedge t) \mathbb{I}_{\{\tau \leqslant t\}} \mid \mathscr{F}_{t}^{\zeta, c}\right]+\mathbb{E}\left[g(\tau \vee t) \mathbb{I}_{\{t<\tau\}} \mid \mathscr{F}_{t}^{\zeta, c}\right]
$$

Now, since $g(\tau \wedge t) \mathbb{I}_{\{\tau \leqslant t\}}$ is $\mathscr{F}_{t}^{\zeta, c}$-measurable, $\mathbb{P}$-a.s, one has

$$
\begin{aligned}
\mathbb{E}\left[g(\tau \wedge t) \mathbb{I}_{\{\tau \leqslant t\}} \mid \mathscr{F}_{t}^{\zeta, c}\right] & =g(\tau \wedge t) \mathbb{I}_{\{\tau \leqslant t\}} \\
& =g(\tau \wedge t) \mathbb{I}_{\left\{\zeta_{t}=1\right\}} .
\end{aligned}
$$


On the other hand, due to the facts that $g(\tau \vee t) \mathbb{I}_{\{t<\tau\}}$ is $\sigma\left(\zeta_{s}, t \leq s \leq+\infty\right) \vee \mathscr{N}_{P^{-}}$ measurable and $\zeta$ is a Markov process with respect to its completed natural filtration we obtain $\mathbb{P}$-a.s.

$$
\mathbb{E}\left[g(\tau \vee t) \mathbb{I}_{\{t<\tau\}} \mid \mathscr{F}_{t}^{\zeta, c}\right]=\mathbb{E}\left[g(\tau \vee t) \mathbb{I}_{\{t<\tau\}} \mid \zeta_{t}\right]
$$

The result is deduced from (17).

(ii) Equation (28) is an immediate consequence of (i). Concerning Eq. (29), we use the same method which we used to prove (i).

Remark 3 The process $\zeta$ cannot be a homogeneous $\mathbb{F}^{\zeta}$-Markov process. Indeed, Proposition 10 enables us to see that, for $A \in \mathscr{B}(\mathbb{R})$ and $t<u$, we have $\mathbb{P}$-a.s.,

$$
\begin{aligned}
\mathbb{P}\left(\zeta_{u} \in A \mid \mathscr{F}_{t}^{\zeta}\right)= & \mathbb{I}_{\{1 \in A\}} \mathbb{I}_{\left\{\zeta_{t}=1\right\}}+\mathbb{I}_{\{1 \in A\}} \int_{(t, u]} \phi_{\zeta_{t}^{r}}\left(\zeta_{t}\right) \mathbb{P}_{\tau}(\mathrm{d} r) \mathbb{I}_{\left\{0<\zeta_{t}<1\right\}} \\
& +\int_{(u,+\infty)} \int_{A} \mathbb{I}_{\left\{\zeta_{t}<y<1\right\}} \mathbb{P}_{\zeta_{u}^{r} \mid \zeta_{t}^{r}=x}(\mathrm{~d} y) \phi_{\zeta_{t}^{r}}\left(\zeta_{t}\right) \mathbb{P}_{\tau}(\mathrm{d} r) \mathbb{I}_{\left\{0<\zeta_{t}<1\right\}},
\end{aligned}
$$

which is clear that it does not depend only on $u-t$.

\subsection{Markov Property with Respect to $\mathbb{F}_{+}^{\zeta, c}$}

We have established, in the previous section, the Markov property of $\zeta$ with respect to its completed natural filtration $\mathbb{F}^{\zeta, c}$. In this section, we are interested in the the Markov property of $\zeta$ with respect to $\mathbb{F}_{+}^{\zeta, c}$. It has an interesting consequence which is none other than the filtration $\mathbb{F}^{\zeta}, c$ satisfies the usual conditions of completeness and right-continuity. However, we need the following condition of on the integrability of $\tau$.

Assumption 2 There exists a sufficiently small $\alpha>0$ such that

$$
\mathbb{E}\left(\tau^{\alpha}\right)<+\infty
$$

The next theorem shows the Markov property of $\zeta$ with respect to $\mathbb{F}_{+}^{\zeta, c}$.

Theorem 2 The process $\zeta$ is a Markov process with respect to $\mathbb{F}_{+}^{\zeta, c}$.

Proof It is sufficient to prove that for any $0 \leq t<u$ and any function bounded continuous $g$, we have

$$
\mathbb{E}\left[g\left(\zeta_{u}\right) \mid \mathscr{F}_{t+}^{\zeta, c}\right]=\mathbb{E}\left[g\left(\zeta_{u}\right) \mid \zeta_{t}\right], \quad \mathbb{P}-a . s
$$

Let $\left(t_{n}\right)_{n \in \mathbb{N}}$ be a decreasing sequence of strictly positive real numbers converging to $t$ : that is $0 \leq t<\cdots<t_{n+1}<t_{n}<\cdots<t_{1}<u, t_{n} \searrow t$ as $n \longrightarrow+\infty$. Since $g$ is 
bounded and $\mathscr{F}_{t+}^{\zeta, c}=\bigcap_{n} \mathscr{F}_{t_{n}}^{\zeta, c}, \mathbb{P}$-a.s., we have

$$
\mathbb{E}\left[g\left(\zeta_{u}\right) \mid \mathscr{F}_{t+}^{\zeta, c}\right]=\lim _{n \longmapsto+\infty} \mathbb{E}\left[g\left(\zeta_{u}\right) \mid \mathscr{F}_{t_{n}}^{\zeta, c}\right]
$$

It follows from the Markov property of $\zeta$ with respect to $\mathbb{F}^{\zeta}, c$ that

$$
\mathbb{E}\left[g\left(\zeta_{u}\right) \mid \mathscr{F}_{t+}^{\zeta, c}\right]=\lim _{n \longmapsto+\infty} \mathbb{E}\left[g\left(\zeta_{u}\right) \mid \zeta_{t_{n}}\right], \quad \mathbb{P} \text {-a.s. }
$$

It remains to prove that

$$
\lim _{n \rightarrow+\infty} \mathbb{E}\left[g\left(\zeta_{u}\right) \mid \zeta_{t_{n}}\right]=\mathbb{E}\left[g\left(\zeta_{u}\right) \mid \zeta_{t}\right], \quad \mathbb{P} \text {-a.s. }
$$

The proof is splitted into two parts. In the first one, we show statement (31) for $t>0$, while in the second part we consider the case $t=0$.

Let $t>0$. We begin by noticing that from Proposition $10, \mathbb{P}$-a.s., we have

$$
\begin{aligned}
\mathbb{E}\left[g\left(\zeta_{u}\right) \mid \zeta_{t_{n}}\right]= & g(1)\left(\mathbb{I}_{\left\{\zeta_{t_{n}}=1\right\}}+\int_{\left(t_{n}, u\right]} \phi_{\zeta_{t_{n}}^{r}}\left(\zeta_{t_{n}}\right) \mathbb{P}_{\tau}(\mathrm{d} r) \mathbb{I}_{\left\{0<\zeta_{t_{n}}<1\right\}}\right) \\
& +\int_{(u,+\infty)} K_{t_{n}, u}\left(r, \zeta_{t_{n}}\right) \phi_{\zeta_{t_{n}}^{r}}\left(\zeta_{t_{n}}\right) \mathbb{P}_{\tau}(\mathrm{d} r) \mathbb{I}_{\left\{0<\zeta_{t_{n}}<1\right\}} \\
= & g(1)\left(\mathbb{I}_{\left\{\tau \leq t_{n}\right\}}+\int_{\left(t_{n}, u\right]} \phi_{\zeta_{t_{n}}^{r}}\left(\zeta_{t_{n}}\right) \mathbb{P}_{\tau}(\mathrm{d} r) \mathbb{I}_{\left\{t_{n}<\tau\right\}}\right) \\
& +\int_{(u,+\infty)} K_{t_{n}, u}\left(r, \zeta_{t_{n}}\right) \phi_{\zeta_{t_{n}}^{r}}\left(\zeta_{t_{n}}\right) \mathbb{P}_{\tau}(\mathrm{d} r) \mathbb{I}_{\left\{t_{n}<\tau\right\}} .
\end{aligned}
$$

Where the function $K_{t, u}(r, x)$ is defined on $\mathbb{R}$ by for $0<t<u<r$

$$
\begin{aligned}
K_{t, u}(r, x) & :=\mathbb{E}\left[g\left(\zeta_{u}^{r}\right) \mid \zeta_{t}^{r}=x\right] \\
& =\int_{\mathbb{R}} g(y) \mathbb{I}_{\{x<y<1\}} \mathbb{P}_{\zeta_{u}^{r} \mid \zeta_{t}^{r}=x}(\mathrm{~d} y) .
\end{aligned}
$$

Since $\lim _{n \longrightarrow+\infty} \mathbb{I}_{\left\{t_{n}<\tau\right\}}=\mathbb{I}_{\{t<\tau\}}$, assertion (34) will be established if we show, $\mathbb{P}$-a.s on $\{t<\tau\}$, that

$$
\lim _{n \longrightarrow+\infty} \int_{\left(t_{n}, u\right]} \phi_{\zeta_{t_{n}}^{r}}\left(\zeta_{t_{n}}\right) \mathbb{P}_{\tau}(\mathrm{d} r)=\int_{(t, u]} \phi_{\zeta_{t}^{r}}\left(\zeta_{t}\right) \mathbb{P}_{\tau}(\mathrm{d} r)
$$

and

$$
\lim _{n \longrightarrow+\infty} \int_{(u,+\infty)} K_{t_{n}, u}\left(r, \zeta_{t_{n}}\right) \phi_{\zeta_{t_{n}}^{r}}\left(\zeta_{t_{n}}\right) \mathbb{P}_{\tau}(\mathrm{d} r)=\int_{(u,+\infty)} K_{t, u}\left(r, \zeta_{t}\right) \phi_{\zeta_{t}^{r}}\left(\zeta_{t}\right) \mathbb{P}_{\tau}(\mathrm{d} r)
$$


We start by proving assertion (36). The integral on the left-hand side of (36) can be rewritten as

$$
\begin{aligned}
\int_{\left(t_{n}, u\right]} \phi_{\zeta_{t_{n}}^{r}}\left(\zeta_{t_{n}}\right) \mathbb{P}_{\tau}(\mathrm{d} r)= & \frac{\int_{\left(t_{n}, u\right]} \varphi_{\zeta_{t_{n}}^{r}}\left(\zeta_{t_{n}}\right) \mathbb{P}_{\tau}(\mathrm{d} r)}{\int_{\left(t_{n},+\infty\right)} \varphi_{\zeta_{t_{n}}^{s}}\left(\zeta_{t_{n}}\right) \mathbb{P}_{\tau}(\mathrm{d} s)} \mathbb{I}_{\left\{0<\zeta_{t_{n}}<1\right\}} \\
= & \frac{\int_{\left(t_{n}, u\right]}\left(1-\zeta_{t_{n}}\right)^{r} \frac{\Gamma(r)}{\Gamma\left(r-t_{n}\right)} \mathbb{P}_{\tau}(\mathrm{d} r)}{\int_{\left(t_{n},+\infty\right)}\left(1-\zeta_{t_{n}}\right)^{s} \frac{\Gamma(s)}{\Gamma\left(s-t_{n}\right)} \mathbb{P}_{\tau}(\mathrm{d} s)} \mathbb{I}_{\left\{0<\zeta_{t_{n}}<1\right\}} .
\end{aligned}
$$

First, let us remark that the function

$$
(t, r, x) \longrightarrow(1-x)^{r} \frac{\Gamma(r)}{\Gamma(r-t)}
$$

defined on $\left\{(t, r) \in(0,+\infty)^{2}, t<r\right\} \times(0,1)$ is continuous. Using the facts that $\zeta_{t_{n}}$ is decreasing to $\zeta_{t}$ and $\mathbb{P}\left[\zeta_{t}=0\right]=0, \mathbb{P}$-a.s on $\{t<\tau\}$, we have

$$
\lim _{n \rightarrow+\infty}\left(1-\zeta_{t_{n}}\right)^{r} \frac{\Gamma(r)}{\Gamma\left(r-t_{n}\right)} \mathbb{I}_{\left\{t_{n}<r\right\}} \mathbb{I}_{\left\{0<\zeta_{t_{n}}<1\right\}}=\left(1-\zeta_{t}\right)^{r} \frac{\Gamma(r)}{\Gamma(r-t)} \mathbb{I}_{\{t<r\}} \mathbb{I}_{\left\{0<\zeta_{t}<1\right\}} .
$$

On the other hand, since the function $x \longmapsto(1-x)^{r}$ is decreasing on $(0,1)$ for all $r>0$ and

$$
0 \leq \frac{\Gamma(r)}{\Gamma(r-t)}=r^{t}\left[1-\frac{t(t+1)}{2 r}+O\left(\frac{1}{r^{2}}\right)\right],
$$

for large enough $r$, see [1], p. 257, 6.1.46, then for any compact subset $\mathscr{K}$ of $(0,+\infty) \times$ $(0,1)$ it yields

$$
\sup _{(t, x) \in \mathscr{K}}(1-x)^{r} \frac{\Gamma(r)}{\Gamma(r-t)} \mathbb{I}_{\{t<r\}}<+\infty .
$$

Hence, $\mathbb{P}$-a.s on $\{t<\tau\}$, we have

$$
\sup _{n \in \mathbb{N}}\left(1-\zeta_{t_{n}}\right)^{r} \frac{\Gamma(r)}{\Gamma\left(r-t_{n}\right)} \mathbb{I}_{\left\{t_{n}<r\right\}} \mathbb{I}_{\left\{0<\zeta_{t_{n}}<1\right\}}<+\infty .
$$

We conclude assertion (36) from the Lebesgue dominated convergence theorem.

Now, let us prove (37). Recall that the function $K_{t_{n}, u}\left(r, \zeta_{t_{n}}\right)$ is given by

$$
\begin{aligned}
& K_{t_{n}, u}\left(r, \zeta_{t_{n}}\right)=\left.\int_{\mathbb{R}} g(y) \mathbb{I}_{\{x<y<1\}} \mathbb{P}_{\zeta_{u}^{r} \mid \zeta_{t_{n}}^{r}=x}(\mathrm{~d} y)\right|_{x=\zeta_{t_{n}}} \\
& =\frac{\Gamma\left(r-t_{n}\right)}{\Gamma\left(u-t_{n}\right) \Gamma(r-u)} \int_{\mathbb{R}} g(y) \frac{\left(y-\zeta_{t_{n}}\right)^{u-t-1}(1-y)^{r-u-1}}{\left(1-\zeta_{t_{n}}\right)^{r-t-1}} \mathbb{I}_{\left\{\zeta_{t_{n}}<y<1\right\}} \mathrm{d} y .
\end{aligned}
$$


Since $g$ is bounded, we deduce that $K_{t_{n}, u}\left(r, \zeta_{t_{n}}\right)$ is bounded. Moreover, we obtain from the weak convergence that

$$
\lim _{n \rightarrow+\infty} K_{t_{n}, u}\left(r, \zeta_{t_{n}}\right)=K_{t, u}\left(r, \zeta_{t}\right)
$$

$\mathbb{P}$-a.s on $\{t<\tau\}$. Combining the fact that $K_{t_{n}, u}\left(r, \zeta_{t_{n}}\right)$ is bounded, (38) and (40) assertion (37) is then derived from the Lebesgue dominated convergence theorem.

Next, we investigate the second part of the proof, that is the case $t=0$. It will be carried out in two steps. In the first one, we assume that there exists $\varepsilon>0$ such that

$$
\mathbb{P}(\tau>\varepsilon)=1
$$

As in the first part, it is sufficient to verify that

$$
\lim _{n \longrightarrow+\infty} \mathbb{E}\left[g\left(\zeta_{u}\right) \mid \zeta_{t_{n}}\right]=\mathbb{E}\left[g\left(\zeta_{u}\right) \mid \zeta_{0}\right], \quad \mathbb{P} \text {-a.s. }
$$

Without loss of generality, we assume $t_{n}<\alpha \wedge \varepsilon$ for all $n \in \mathbb{N}$. It is easy to see that under condition (41), $\mathbb{E}\left[g\left(\zeta_{u}\right) \mid \zeta_{t_{n}}\right]$ takes the form

$$
\begin{aligned}
\mathbb{E}\left[g\left(\zeta_{u}\right) \mid \zeta_{t_{n}}\right]= & g(1) \int_{(\varepsilon, u]} \phi_{\zeta_{t_{n}}^{r}}\left(\zeta_{t_{n}}\right) \mathbb{P}_{\tau}(\mathrm{d} r) \\
& +\int_{(u,+\infty)} K_{t_{n}, u}\left(r, \zeta_{t_{n}}\right) \phi_{\zeta_{t_{n}}^{r}}\left(\zeta_{t_{n}}\right) \mathbb{P}_{\tau}(\mathrm{d} r)
\end{aligned}
$$

On the other hand, we have

$$
\begin{aligned}
\mathbb{E}\left[g\left(\zeta_{u}\right) \mid \zeta_{0}\right]= & \mathbb{E}\left[g\left(\zeta_{u}\right)\right]=g(1) F(u) \\
& +\int_{(u,+\infty)} \int_{\mathbb{R}} g(y) \varphi_{\zeta_{t}^{r}}(y) \mathrm{d} y \mathbb{P}_{\tau}(\mathrm{d} r) .
\end{aligned}
$$

Then, in order to show (42) it is sufficient to prove, $\mathbb{P}$-a.s, the following

$$
\lim _{n \longrightarrow+\infty} \int_{(\varepsilon, u]} \phi_{\zeta_{t_{n}}^{r}}\left(\zeta_{t_{n}}\right) \mathbb{P}_{\tau}(\mathrm{d} r)=F(u),
$$

and

$$
\begin{aligned}
& \lim _{n \rightarrow+\infty} \int_{(u,+\infty)} K_{t_{n}, u}\left(r, \zeta_{t_{n}}\right) \phi_{\zeta_{t_{n}}^{r}}\left(\zeta_{t_{n}}\right) \mathbb{P}_{\tau}(\mathrm{d} r) \\
& \quad=\int_{(u,+\infty)} \int_{\mathbb{R}} g(y) \varphi_{\zeta_{t}^{r}}(y) \mathrm{d} y \mathbb{P}_{\tau}(\mathrm{d} r)
\end{aligned}
$$


First, for $r>\varepsilon$, we have

$$
\lim _{n \rightarrow+\infty}\left(1-\zeta_{t_{n}}\right)^{r} \frac{\Gamma(r)}{\Gamma\left(r-t_{n}\right)} \mathbb{I}_{\left\{t_{n}<r\right\}} \mathbb{I}_{\left\{0<\zeta_{t_{n}}<1\right\}}=1
$$

Since the gamma function is increasing on $[2, \infty)$, for $r \geq 2+t_{1}$, we obtain

$$
\sup _{n \in \mathbb{N}}\left(1-\zeta_{t_{n}}\right)^{r} \frac{\Gamma(r)}{\Gamma\left(r-t_{n}\right)}<\frac{\Gamma(r)}{\Gamma\left(r-t_{1}\right)}
$$

It follows from (30) and (39) that the function $r \longmapsto \frac{\Gamma(r)}{\Gamma\left(r-t_{1}\right)}$ is $\mathbb{P}_{\tau}$-integrable on $(\varepsilon,+\infty)$. Hence, (43) follows from a simple application of the Lebesgue dominated convergence theorem. In the same way as in the first case $(t>0)$, we obtain from the weak convergence that

$$
\lim _{n \rightarrow+\infty} K_{t_{n}, u}\left(r, \zeta_{t_{n}}\right)=\frac{\Gamma(r)}{\Gamma(u) \Gamma(r-u)} \int_{\mathbb{R}} g(y) y^{u-1}(1-y)^{r-u-1} \mathbb{I}_{\{0<y<1\}} \mathrm{d} y,
$$

then also (44) follows from a simple application of the Lebesgue dominated convergence theorem. Finally, we have to consider the general case, that is $\mathbb{P}(\tau>0)=1$. In order to prove the Markov property of $\zeta$ with respect to $\mathbb{F}_{+}^{\zeta, c}$ at $t=0$, it is suffi-

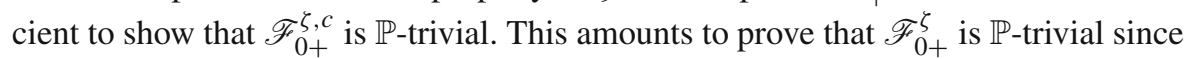
$\mathscr{F}_{0+}^{\zeta, c}=\mathscr{F}_{0+}^{\zeta} \vee \mathscr{N}_{P}$. To do so, let $\varepsilon>0$ be fixed and consider the stopping time $\tau_{\varepsilon}=\tau \vee \varepsilon$. We define the process $\zeta_{t}^{\tau_{\varepsilon}}$ by

$$
\left\{\zeta_{t}^{\tau_{\varepsilon}} ; t \geq 0\right\}:=\left\{\left.\zeta_{t}^{r}\right|_{r=\tau \vee \varepsilon} ; t \geq 0\right\}
$$

The first remark is that the sets $\left(\tau_{\varepsilon}>\varepsilon\right)=(\tau>\varepsilon)$ are equal and therefore the following equality of processes holds

$$
\zeta^{\tau_{\varepsilon}} \mathbb{I}_{(\tau>\varepsilon)}=\zeta . \mathbb{I}_{(\tau>\varepsilon)}
$$

Then, for each $A \in \mathscr{F}_{0+}^{\zeta}$ there exists $B \in \mathscr{F}_{0+}^{\zeta^{\tau_{\varepsilon}}}$ such that

$$
A \cap(\tau>\varepsilon)=B \cap(\tau>\varepsilon) .
$$

As $\mathbb{P}\left(\tau_{\varepsilon}>\varepsilon / 2\right)=1$, according to the previous case we have that $\mathscr{F}_{0+}^{\zeta^{\tau_{\varepsilon}}}$ is $\mathbb{P}$-trivial. That is, $\mathbb{P}(B)=0$ or 1 . Consequently, we obtain

$$
\mathbb{P}(A \cap(\tau>\varepsilon))=0 \text { or } \mathbb{P}(A \cap(\tau>\varepsilon))=\mathbb{P}(\tau>\varepsilon) .
$$

Now if $\mathbb{P}(A)>0$, then there exists $\varepsilon>0$ such that $\mathbb{P}(A \cap\{\tau>\varepsilon\})>0$. Therefore, for all $0<\varepsilon^{\prime} \leq \varepsilon$ we have

$$
\mathbb{P}\left(A \cap\left(\tau>\varepsilon^{\prime}\right)\right)=\mathbb{P}\left(\tau>\varepsilon^{\prime}\right) .
$$


Passing to the limit as $\varepsilon^{\prime}$ goes to 0 yields $\mathbb{P}(A \cap(\tau>0))=\mathbb{P}(\tau>0)=1$. It follows that $\mathbb{P}(A)=1$, which ends the proof.

Corollary 4 The filtration $\mathbb{F}^{\zeta, c}$ satisfies the usual conditions of right-continuity and completeness.

Proof See, e.g. [[6], Ch. I, Proposition (8.12)]

\subsection{Semi-martingale Decomposition of $\zeta$}

Our purpose is to derive the semi-martingale property of $\zeta$ with respect to its own filtration $\mathbb{F}^{\zeta, c}$. Firstly, we obtain from representation (15) that

$$
\zeta_{t}=\widehat{N}_{t}+\int_{0}^{t} Z_{s} \mathrm{~d} s, \quad t \geq 0,
$$

where the processes $\widehat{N}$ and $Z$ are defined as follows:

$$
\widehat{N}_{t}(\omega):=\left.\widehat{N}_{t}^{r}(\omega)\right|_{r=\tau(\omega)},
$$

and

$$
Z_{t}=\frac{1-\zeta_{t}}{\tau-t} \mathbb{I}_{\{t<\tau\}}
$$

for $(t, \omega) \in \mathbb{R}_{+} \times \Omega$. Now, let us consider the filtration

$$
\mathbb{H}=\left(\mathscr{H}_{t}:=\mathscr{F}_{t}^{\zeta, c} \vee \sigma(\tau), \quad t \geq 0\right)
$$

which is equal to the initial enlargement of the filtration $\mathbb{F}^{\zeta, c}$ by the $\sigma$-algebra $\sigma(\tau)$. Since the processes $\zeta$ and $Z$ are $\mathbb{H}$-adapted, it follows from equation (46) that $\widehat{N}$ is $\mathbb{H}$-adapted. Moreover, $\tau$ is a stopping time with respect to $\mathbb{H}$. The next proposition will play a very important role in forthcoming developments, since it shows the semimartingale property of $\zeta$ with respect to $\mathbb{H}$.

Proposition 11 (i) We have

$$
\mathbb{E}\left[\int_{0}^{t}\left|Z_{s}\right| d s\right]<+\infty, \forall t \geq 0 .
$$

(ii) The process $\widehat{N}=\left(\widehat{N}_{t}, t \geq 0\right)$ defined by

$$
\widehat{N}_{t}=\zeta_{t}-\int_{0}^{t} Z_{s} d s, \quad t \geq 0,
$$

is a $\mathbb{H}$-martingale stopped at $\tau$.

Proof (i) We first note that $Z$ is a non-negative process. Since $s \leq r, \zeta_{s}^{r}$ has a beta distribution $\beta(s, r-s), \mathbb{E}\left(\zeta_{s}^{r}\right)=s / r$. So, we can see, for any $t \geq 0$, that 


$$
\begin{aligned}
\mathbb{E}\left[\int_{0}^{t} Z_{s} \mathrm{~d} s\right] & =\int_{0}^{+\infty} \int_{0}^{t \wedge r} \frac{1-\mathbb{E}\left[\zeta_{s}^{r}\right]}{r-s} \mathrm{~d} s \mathbb{P}_{\tau}(\mathrm{d} r) \\
& =\int_{0}^{+\infty} \int_{0}^{t \wedge r} \frac{1}{r} \mathrm{~d} s \mathbb{P}_{\tau}(\mathrm{d} r) \leq 1 .
\end{aligned}
$$

(ii) By assertion (i), the process $\left(Z_{t}, t \geq 0\right)$ is integrable with respect to the Lebesgue measure, hence $\widehat{N}$ is well defined. It is clear that the process $\widehat{N}$ is $\mathbb{H}$-adapted and $\widehat{N}_{t}=\widehat{N}_{\tau}, \mathbb{P}$-a.s, on the set $\{t \geq \tau\}$. Now, since $\left(\widehat{N}_{t}^{r}, t \geq 0\right)$ is a $\mathbb{F}^{\zeta} \zeta^{r}$-martingale stopped at $r$ we obtain, for any $0<t_{1}<t_{2}<\cdots<t_{n}=t, n \in \mathbb{N}^{*}, h \geq 0$ and $g$ a bounded Borel function, that

$$
\begin{aligned}
\mathbb{E} & {\left[\left(\widehat{N}_{t+h}-\widehat{N}_{t}\right) g\left(\zeta_{t_{1}}, \ldots, \zeta_{t_{n}}, \tau\right)\right] } \\
= & \int_{(0,+\infty)} \mathbb{E}\left[\left(\widehat{N}_{t+h}^{r}-\widehat{N}_{t}^{r}\right) g\left(\zeta_{t_{1}}^{r}, \ldots, \zeta_{t_{n}}^{r}, r\right)\right] \mathbb{P}_{\tau}(\mathrm{d} r) \\
= & \int_{(0, t)} \mathbb{E}\left[\left(\widehat{N}_{t+h}^{r}-\widehat{N}_{t}^{r}\right) g\left(\zeta_{t_{1}}^{r}, \ldots, \zeta_{t_{n}}^{r}, r\right)\right] \mathbb{P}_{\tau}(\mathrm{d} r) \\
& +\int_{[t, t+h)} \mathbb{E}\left[\left(\widehat{N}_{t+h}^{r}-\widehat{N}_{t}^{r}\right) g\left(\zeta_{t_{1}}^{r}, \ldots, \zeta_{t_{n}}^{r}, r\right)\right] \mathbb{P}_{\tau}(\mathrm{d} r) \\
& +\int_{[t+h,+\infty)} \mathbb{E}\left[\left(\widehat{N}_{t+h}^{r}-\widehat{N}_{t}^{r}\right) g\left(\zeta_{t_{1}}^{r}, \ldots, \zeta_{t_{n}}^{r}, r\right)\right] \mathbb{P}_{\tau}(\mathrm{d} r) \\
= & \int_{(0, t)} \mathbb{E}\left[\left(\widehat{N}_{r}^{r}-\widehat{N}_{r}^{r}\right) g\left(\zeta_{t_{1}}^{r}, \ldots, \zeta_{t_{n}}^{r}, r\right)\right] \mathbb{P}_{\tau}(\mathrm{d} r) \\
& +\int_{[t, t+h)} \mathbb{E}\left[\left(\widehat{N}_{r}^{r}-\widehat{N}_{t}^{r}\right) g\left(\zeta_{t_{1}}^{r}, \ldots, \zeta_{t_{n}}^{r}, r\right)\right] \mathbb{P}_{\tau}(\mathrm{d} r) \\
& +\int_{[t+h,+\infty)} \mathbb{E}\left[\left(\widehat{N}_{t+h}^{r}-\widehat{N}_{t}^{r}\right) g\left(\zeta_{t_{1}}^{r}, \ldots, \zeta_{t_{n}}^{r}, r\right)\right] \mathbb{P}_{\tau}(\mathrm{d} r)=0 .
\end{aligned}
$$

The desired result follows by a standard monotone class argument. This completes the proof.

Therefore, it follows from Stricker's Theorem [24] that $\zeta$ is a semi-martingale relative to its natural filtration $\mathbb{F}^{\zeta, c}$. A natural question is: What is the explicit form of its canonical decomposition? That is the problem we want to discuss. The method consists in applying the stochastic filtering theory.

Theorem 3 The canonical decomposition of $\zeta$ in its natural filtration $\mathbb{F}^{\zeta, c}$ is given by

$$
\zeta_{t}=\tilde{N}_{t}+\int_{0}^{t}\left(1-\zeta_{s}\right) \int_{(s,+\infty)} \frac{1}{r-s} \phi_{\zeta_{s}^{r}}\left(\zeta_{s}\right) \mathbb{P}_{\tau}(d r) \mathbb{I}_{\left\{0<\zeta_{s}<1\right\}} d s,
$$

where $\left(\widetilde{N}_{t}, t \geq 0\right)$ is an $\mathbb{F}^{\zeta, c}$-martingale stopped at $\tau$.

Proof Let us start by recalling that $\tau$ is a stopping time with respect to $\mathbb{F}^{\zeta, c}$. A wellknown result of filtering theory [7] (T1, p. 87) (or Theorem 8.1.1 and Remark 8.1.1 
[17] for more general setting) tells us that the decomposition of $\zeta$ in its natural filtration $\mathbb{F}^{\zeta, c}$ is given by

$$
\zeta_{t}=\widetilde{N}_{t}+\int_{0}^{t} \mathbb{E}\left(Z_{s} \mid \mathscr{F}_{s}^{\zeta, c}\right) \mathrm{d} s,
$$

where $\left(\tilde{N}_{t}, t \geq 0\right)$ is an $\mathbb{F}^{\zeta, c}$-martingale stopped at $\tau$. Therefore, we have only to compute the conditional expectation of $Z_{S}$ relative to $\mathscr{F}_{S}^{\zeta, c}$. Indeed, using (27) we have

$$
\begin{aligned}
\mathbb{E}\left(Z_{S} \mid \mathscr{F}_{s}^{\zeta, c}\right) & =\mathbb{E}\left(\frac{1-\zeta_{s}}{\tau-s} \mathbb{I}_{\{s<\tau\}} \mid \mathscr{F}_{S}^{\zeta, c}\right)=\left(1-\zeta_{S}\right) \mathbb{E}\left(\frac{1}{\tau-s} \mathbb{I}_{\{s<\tau\}} \mid \mathscr{F}_{s}^{\zeta, c}\right) \\
& =\left(1-\zeta_{s}\right) \int_{(s,+\infty)} \frac{1}{r-s} \phi_{\zeta_{s}^{r}}\left(\zeta_{s}\right) \mathbb{P}_{\tau}(\mathrm{d} r) \mathbb{I}_{\left\{0<\zeta_{s}<1\right\}} .
\end{aligned}
$$

Hence, we derive the canonical decomposition (49) of $\zeta$ as a semi-martingale in its own filtration $\mathbb{F}^{\zeta, c}$.

Remark 4 The results of the paper can be straightforwardly extended to a large class of gamma subordinator $\left(\gamma_{t}^{(\eta, \kappa)}, t \geq 0\right), \eta, \kappa>0$, with Lévy measure

$$
v(\mathrm{~d} x)=\frac{\kappa}{x} \exp (-\eta x) \mathbb{I}_{(0, \infty)}(x) \mathrm{d} x
$$

and whose law at time $t$ is the gamma distribution with density

$$
f_{\gamma_{t}^{(\eta, \kappa)}}(x)=\frac{\eta^{\kappa t} x^{\kappa t-1} \exp (-\eta x)}{\Gamma(\kappa t)} \mathbb{I}_{(0, \infty)}(x) .
$$

The Lévy-Khintchine representation is given by

$$
\mathbb{E}\left(\exp \left(-\lambda \gamma_{t}^{(\eta, \kappa)}\right)\right)=\left(1+\frac{\lambda}{\eta}\right)^{-\kappa t} .
$$

On the other hand, they can be also easily extended to the gamma bridges of length $r$, starting at 0 , with an arbitrary ending point $a>0$

$$
\zeta_{t}^{r}:=a \frac{\gamma_{t \wedge r}^{(m)}}{\gamma_{r}^{(m)}}, \quad t \geq 0 .
$$

For the sake of simplicity, we have therefore considered only the case $\eta=\kappa=a=1$ without loss of generality.

Acknowledgements Open access funding provided by Linnaeus University. The authors would like to express particular thanks to the anonymous referee for the constructive comments, and Youssef Ouknine for his guidance and encouragement. The third author gratefully acknowledges the financial support by an Erasmus+ International Credit Mobility exchange project coordinated by Linnaeus University.

Open Access This article is distributed under the terms of the Creative Commons Attribution 4.0 International License (http://creativecommons.org/licenses/by/4.0/), which permits unrestricted use, distribution, and reproduction in any medium, provided you give appropriate credit to the original author(s) and the source, provide a link to the Creative Commons license, and indicate if changes were made. 


\section{References}

1. Abramowitz, M., Stegun, I.A. (eds.): Handbook of Mathematical Functions with Formulas, Graphs, and Mathematical Tables. National Bureau of Standards, Applied Mathematics Series 55, 9th printing, Washington (1970)

2. Asmussen, S., Hobolth, A.: Markov bridges, bisection and variance reduction. In: Plaskota, L., Wozniakowski, H. (eds.) Monte Carlo and Quasi-Monte Carlo Methods 2010. Springer Proceedings in Mathematics and Statistics, vol. 23. Springer, Berlin (2012)

3. Bedini, M.L.: Information on a Default Time: Brownian Bridges on Stochastic Intervals and Enlargement of Filtrations. PhD Thesis, Friedrich Schiller University of Jena (Germany) (2012)

4. Bertoin, J.: Lévy Processes. Cambridge University Press, Cambridge (1996)

5. Bedini, M.L., Buckdahn, R., Engelbert, H.J.: Brownian bridges on random intervals. Theory Probab. Appl. 61(1), 15-39 (2017)

6. Blumenthal, R.M., Getoor, R.K.: Markov Processes and Potential Theory. Academic Press, New York (1968)

7. Brèmaud, P.: Point Processes and Queues. Springer Series in Statistics. Springer, New York (1981)

8. Brody, D.C., Hughston, L.P., Macrina, A.: Dam rain and cumulative gain. Proc. R. Soc. A 464, 1801$1822(2008)$

9. Dufresne, F., Gerber, H.U., Shiu, E.S.: Risk theory with the gamma process. ASTIN Bull. 21, 177-192 (1991)

10. Chan, Y., Talburt, J., Talley, T.: Data Engineering: Mining, Information and Intelligence. Springer, Norwell (2010)

11. Erraoui, M., Louriki, M.: Bridges with random length: Gaussian-Markovian case. Markov Process. Relat. Fields 4, 669-693 (2018)

12. Emery, M., Yor, M.: A parallel between Brownian bridges and gamma bridges. Publ. RIMS Kyoto Univ. 40(3), 669-688 (2004)

13. Gani, J.: Problems in the probability theory of storage systems. J. R. Stat. Soc. Ser. B. 19, 181-206 (1957)

14. Hammersley, J.M.: Storage problems. Math. Ann. 128, 475-478 (1955)

15. Hoyle, E., Hughston, L.P., Macrina, A.: Stable- $\frac{1}{2}$ bridges and insurance. In: Palczewski, A., Stettner, L. (eds.) Advances in Mathematics of Finance. Banach Center Publications, vol. 104, pp. 95-120. Institute of Mathematics of the Polish Academy of Sciences, Warsaw (2015)

16. Hoyle, E., Mengütürk, L.A.: Archimedean survival processes. J. Multivar. Anal. 115, 1-15 (2013)

17. Kallianpur, G.: Stochastic Filtering Theory. Applications of Mathematics, vol. 13. Springer, New York (1980)

18. Kendall, D.G.: Some problems in the theory of dams. J. R. Stat. Soc. Ser. B 19, 207-212 (1957)

19. Kingman, J.F.C.: On continuous time models in the theory of dams. J. Aust. Math. Soc. 3, 480-487 (1963)

20. Kyprianou, A.E.: Introductory Lectures on Fluctuations of Lévy Processes with Applications. Springer, Berlin (2006)

21. Moran, P.A.P.: A probability theory of a dam with a continuous release. Q. J. Math. Oxf. Ser. 7(1), 130-137 (1956)

22. Sato, K.I.: Lévy Processes and Infinitely Divisible Distributions. Cambridge University Press, Cambridge (1999)

23. Shiryaev, A.N.: Probability. 1, 3rd edn. Springer, New York (2016)

24. Stricker, C.: Quasimartingales, martingales locales, semimartingales et filtration naturelle. Z. Wahrscheinlichkeitstheorie und Verw. Gebiete 39(1), 55-63 (1977)

25. Yor, M.: Some remarkable properties of gamma processes. In: Fu, M.C., Jarrow, R.A., Yen, J.Y.J., Elliott, R.J. (eds.) Advances in Mathematical Finance. Applied and Numerical Harmonic Analysis, pp. 37-47. Birkhäuser, Boston (2007)

Publisher's Note Springer Nature remains neutral with regard to jurisdictional claims in published maps and institutional affiliations. 